\title{
openheart Role for machine learning in sex-specific prediction of successful electrical cardioversion in atrial fibrillation?
}

\author{
Nicklas Vinter (10 , ${ }^{1,2}$ Anne Sofie Frederiksen, ${ }^{3}$ Andi Eie Albertsen, ${ }^{3}$ \\ Gregory Y H Lip, ${ }^{4}$ Morten Fenger-Grøn, ${ }^{5}$ Ludovic Trinquart, ${ }^{6}$ Lars Frost, ${ }^{1,2}$ \\ Dorthe Svenstrup Møller ${ }^{3}$
}

\begin{abstract}
- Additional material is published online only. To view please visit the journal online (http://dx.doi.org/10.1136/ openhrt-2020-001297).
\end{abstract}

To cite: Vinter N, Frederiksen AS, Albertsen AE, et al. Role for machine learning in sex-specific prediction of successful electrical cardioversion in atrial fibrillation?. Open Heart 2020;7:e001297. doi:10.1136/ openhrt-2020-001297

Received 27 March 2020 Revised 11 May 2020 Accepted 11 May 2020

Check for updates

(c) Author(s) (or their employer(s)) 2020. Re-use permitted under CC BY-NC. No commercial re-use. See rights and permissions. Published by BMJ.

${ }^{1}$ Diagnostic Centre, Regionshospitalet Silkeborg, Silkeborg, Denmark 2Department of Clinical Medicine, Aarhus Universitet, Aarhus, Denmark

${ }^{3}$ Department of Cardiology, Viborg Regional Hospital, Viborg, Denmark

${ }^{4}$ Liverpool Centre for Cardiovascular Science, University of Liverpool, Liverpool, UK

${ }^{5}$ Research Unit for General Practice and Department of Public Health, Aarhus University, Aarhus C, Denmark ${ }^{6}$ Department of Biostatistics, Boston University, Boston, Massachusetts, USA

Correspondence to Dr Nicklas Vinter; nicvin@rm.dk

\section{ABSTRACT}

Objective Electrical cardioversion is frequently performed to restore sinus rhythm in patients with persistent atrial fibrillation (AF). However, AF recurs in many patients and identifying the patients who benefit from electrical cardioversion is difficult. The objective was to develop sex-specific prediction models for successful electrical cardioversion and assess the potential of machine learning methods in comparison with traditional logistic regression. Methods In a retrospective cohort study, we examined several candidate predictors, including comorbidities, biochemistry, echocardiographic data, and medication. The outcome was successful cardioversion, defined as normal sinus rhythm immediately after the electrical cardioversion and no documented recurrence of AF within 3 months after. We used random forest and logistic regression models for sex-specific prediction.

Results The cohort comprised 332 female and 790 male patients with persistent AF who underwent electrical cardioversion. Cardioversion was successful in $44.9 \%$ of the women and $49.9 \%$ of the men. The prediction errors of the models were high for both women $(41.0 \%$ for machine learning and $48.8 \%$ for logistic regression) and men $(46.0 \%$ for machine learning and $44.8 \%$ for logistic regression). Discrimination was modest for both machine learning ( 0.59 for women and 0.56 for men) and logistic regression models ( 0.60 for women and 0.59 for men), although the models were well calibrated.

Conclusions Sex-specific machine learning and logistic regression models showed modest predictive performance for successful electrical cardioversion. Identifying patients who will benefit from cardioversion remains challenging in clinical practice. The high recurrence rate calls for thoroughly informed shared decision-making for electrical cardioversion.

\section{INTRODUCTION}

Atrial fibrillation (AF) is the most common sustained cardiac arrhythmia encountered in clinical practice. Electrical cardioversion is frequently performed to restore sinus rhythm and relieve symptoms in patients with persistent AF. Despite high rates of initial success of electrical cardioversion, more than half

\section{Key questions}

What is already known about this subject?

- No prediction tools that identifies patients with atrial fibrillation (AF) who may benefit from electrical cardioversion have been implemented in clinical practice. Accumulating evidence indicates that there are considerable sex differences in the epidemiology of AF. However, previous analyses have not accounted for the potential sex differences in prediction models.

What does this study add?

- Our sex-specific prediction models based on machine learning and logistic regression demonstrated different clinically important predictors between sexes, but the models did not demonstrate improved predictive ability compared with other existing models.

How might this impact on clinical practice?

- Identifying the patients who will benefit from cardioversion is a challenge in clinical practice. The high recurrence rate calls for thoroughly informed shared decision-making for electrical cardioversion.

of the patients may have recurrence of $\mathrm{AF}$ within 1 year. $^{12}$

Electrical cardioversion is resource demanding and carries risks of thromboembolism and anaesthesia-related complications, and therefore, development of prediction models for successful cardioversion would be useful to inform shared decisions. Several characteristics have been identified as predictors of AF recurrence, such as high age, female sex, long duration of AF, heart failure, large left atrial size, hypertension, elevated body mass index, ischaemic heart disease, and chronic kidney disease. ${ }^{2-4}$ However, the evidence of identification of patients who will benefit from cardioversion is weak and prediction of success is difficult in clinical practice. 
Accumulating evidence indicates that there are considerable sex differences in the epidemiology of AF, presentation of $\mathrm{AF}$, prognosis and in the response to antiarrhythmic therapy and radiofrequency ablation (RFA).$^{5-8}$ As the existing literature has examined 'general' predictors of successful cardioversion in cohorts including both sexes, the analyses have not accounted for the potential sex differences in prediction models. Development of separate models in women and men may be warranted to improve predictive accuracy. However, traditional regression approaches do not accommodate complex interaction between patient characteristics, but machine learning methods may be a promising new approach that gains acceptance in cardiovascular medicine. ${ }^{9-11}$

Among patients with persistent AF, we aimed to develop sex-specific prediction models for successful electrical cardioversion and assess the potential of machine learning methods in comparison with traditional logistic regression.

\section{METHODS}

\section{Study population}

We conducted a retrospective cohort study of consecutive patients diagnosed with persistent $\mathrm{AF}$ whose ongoing $\mathrm{AF}$ lasted for more than 48 hours or was of unknown duration. The patients were included with their first electrical cardioversion at Regional Hospital Central Jutland (Viborg Regional Hospital and Silkeborg Regional Hospital) in Denmark from August 2011 through March 2016. No exclusion criteria were applied. The criteria of 48 hours/unknown duration were applied because this cohort was originally established to examine use of oral anticoagulation and waiting time to electrical cardioversion. ${ }^{1}$ From electronic medical records, we systematically collected clinical and laboratory data and information on medical history. The study population comprised both patients with a history of cardioversion and patients who underwent their first cardioversion ever. The patients were followed in a structured multidisciplinary AF clinic and were treated with anticoagulation in accordance with the European clinical guidelines. ${ }^{12}$ Synchronised direct current electrical cardioversions were performed with paddles in anterior-posterior or anterior-lateral position. Energy levels ranged from 100 to $360 \mathrm{~J}$ using a biphasic defibrillator.

\section{Candidate predictors}

We used existing literature to select candidate predictors of successful cardioversion (table 1). ${ }^{2-4}$ We assessed all information on potential predictors before the cardioversion procedure. Heart rate was measured on the date of cardioversion. The definition of excessive use of alcohol was more than eight drinks per week. Hypertension was defined as a history of hypertension or ongoing antihypertensive medication, diabetes mellitus as haemoglobin A1c level $\geq 48 \mathrm{mmol} / \mathrm{mol}$ or ongoing antidiabetic medication, and chronic obstructive pulmonary disease (COPD) as a
Table 1 Baseline characteristics

\begin{tabular}{llc}
\hline & Women $\mathbf{n = 3 3 2}$ & Men $\mathbf{n = 7 9 0}$ \\
\hline Age, years & $71.0(65.0-77.8)$ & $67.0(60.0-73.0)$ \\
\hline Body mass index, $\mathrm{kg} / \mathrm{m}^{2}$ & $27.0(24.1-31.8)$ & $27.8(25.2-31.5)$ \\
\hline Heart rate, $\mathrm{min}^{-1}$ & $93.0(79.8-114.0)$ & $91.0(74.0-115.0)$ \\
\hline Alcohol overuse, $\mathrm{n}(\%)$ & $23(6.9)$ & $110(13.9)$ \\
$\begin{array}{l}\text { Prior cardioversion and/or RFA, } \\
\mathrm{n}(\%)\end{array}$ & $99(29.8)$ & $292(36.9)$ \\
\hline
\end{tabular}

Comorbidity, $\mathrm{n}(\%)$

\begin{tabular}{|c|c|c|}
\hline Hypertension & $195(58.7)$ & $481(60.9)$ \\
\hline Diabetes & $28(8.4)$ & $114(14.4)$ \\
\hline COPD & $58(17.9)$ & 78 (10.1) \\
\hline \multicolumn{3}{|l|}{ Clinical scores, n (\%) } \\
\hline \multicolumn{3}{|l|}{$\mathrm{CHA}_{2} \mathrm{DS}_{2}$-VASC } \\
\hline 0 & $23(6.9)$ & $83(10.5)$ \\
\hline 1 & $10(3.0)$ & $200(25.3)$ \\
\hline$\geq 2$ & $299(90.1)$ & $507(64.2)$ \\
\hline \multicolumn{3}{|l|}{ HAS-BLED } \\
\hline 0 & $63(19.0)$ & $299(29.0)$ \\
\hline 1 & $192(57.8)$ & $360(45.6)$ \\
\hline$\geq 2$ & 77 (23.2) & $201(25.4)$ \\
\hline \multicolumn{3}{|l|}{ Blood tests } \\
\hline $\begin{array}{l}\text { Thyroid-stimulating } \\
\text { hormone, IU/L }\end{array}$ & $1.5(0.9-2.4)$ & $1.6(1.1-2.4)$ \\
\hline Haemoglobin, $g / L$ & $136.9(127.2-148.1)$ & $148.1(136.9-156.2)$ \\
\hline eGFR, $\mathrm{mL} / \mathrm{min} / 1.73 \mathrm{~m}^{2}$ & $66.0(54.0-81.0)$ & $72.0(61.0-84.0)$ \\
\hline \multicolumn{3}{|l|}{ Echocardiographic data } \\
\hline \multicolumn{3}{|c|}{ Left ventricular ejection fraction, $\mathrm{n}(\%)$} \\
\hline$\geq 40 \%$ & $280(84.6)$ & $592(75.0)$ \\
\hline$<40 \%$ & $51(15.4)$ & $197(25.0)$ \\
\hline Left atrial diameter, $\mathrm{cm}$ & $4.2(3.8-4.5)$ & $4.3(4.0-4.7)$ \\
\hline \multicolumn{3}{|l|}{ Medication, n (\%) } \\
\hline \multicolumn{3}{|l|}{ Antiarrhythmic drugs } \\
\hline Class Ic & $11(3.4)$ & $27(3.5)$ \\
\hline Class III & $61(18.8)$ & $142(18.3)$ \\
\hline Beta-blocker & $262(80.6)$ & $573(73.9)$ \\
\hline $\begin{array}{l}\text { Non-dihydropyridine } \\
\text { channel blocker }\end{array}$ & $16(4.9)$ & $50(6.5)$ \\
\hline Digoxin & $90(27.8)$ & $124(16.0)$ \\
\hline ACEI or ARB & $148(45.5)$ & $392(50.6)$ \\
\hline
\end{tabular}

Data are median (25\%-75\% percentiles) or counts (percentages). Missing data: body mass index $0.5 \%$; heart rate $1.0 \%$; chronic obstructive pulmonary disease (COPD) $2.0 \%$; thyroid-stimulating hormone 5.9\%; haemoglobin 1.0\%; estimated glomerular filtration rate (eGFR) $0.9 \%$; left ventricular ejection fraction $0.2 \%$; left atrial diameter 2.7\%; antiarrhythmic drugs 2.0\%; beta-blocker 2.0\%; nondihydropyridine channel blockers, $2.0 \%$; digoxin $2.0 \%$; ACE inhibitor (ACEI) 2.0\%; angiotensin II receptor blocker (ARB) 2.7\%. CHA2DS2-VASc: Congestive heart failure, hypertension, age $\geq 75$ years, diabetes mellitus, prior stroke, tia or thromboembolism, vascular disease, age 65-74 years, and female sex. HAS-BLED: Hypertension, abnormal renal or liver function, prior history of stroke, prior major bleeding, labile INR, age>65 years, and drugs or alcohol.

RFA, radiofrequency ablation. 

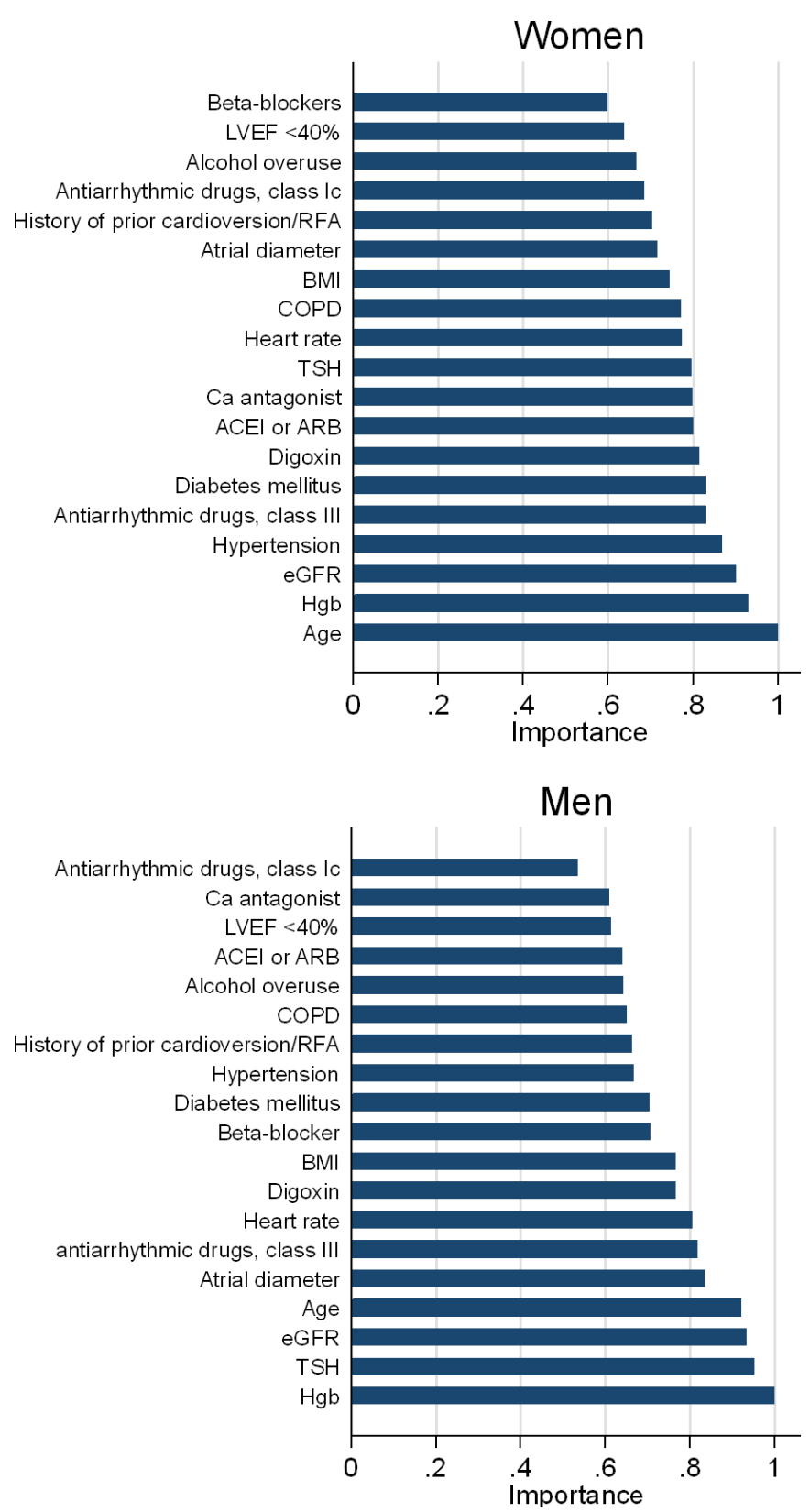

Figure 1 Importance score of predictor variables by sex. ACEI, ACE inhibitor; ARB, angiotensin II receptor blocker; BMI, body mass index; COPD, chronic obstructive pulmonary disease; eGFR, estimated glomerular filtration rate; Hgb, haemoglobin; LVEF, left ventricular ejection fraction; RFA, radiofrequency ablation; TSH, thyroidstimulating hormone.

pulmonary function test with irreversible airflow limitation, expressed as a FEV1/FVC ratio $<0.7$ after bronchodilatation, or ongoing inhalation treatment consistent with COPD. Echocardiographic data included left ventricular ejection fraction and left atrial diameter measured from the parasternal long axis. Echocardiograms were considered valid if the examination was performed within a period of 3 months before the cardioversion.

\section{Outcome}

The outcome was successful electrical cardioversion, defined as sinus rhythm immediately after the cardioversion and no documented recurrence of $\mathrm{AF}$ within 3 months. Following cardioversion, all patients were examined with telemetry for at least 2 hours. Afterwards, the patients could report recurrence of symptoms to the hospital that led to examination for AF. Over the 3-month follow-up, AF could be detected using ECG or Holter monitoring by clinical indication. At 3 months, all patients went to a control examination, which included examination with ECG.

\section{Statistical methods}

We performed all analyses in women and men separately.

We considered the following continuous predictors: age, body mass index, heart rate, thyroid-stimulating hormone (TSH) level, haemoglobin, estimated glomerular filtration rate (eGFR), and left atrial diameter. We also considered the following categorical predictors: alcohol use, history of prior cardioversion and/or RFA, hypertension, diabetes, COPD, low left ventricular ejection fraction $(<40 \%)$, use of antiarrhythmic drugs, use of beta-blockers, use of non-dihydropyridine calcium channel blockers, use of digoxin, use of ACE inhibitor (ACEI), or angiotensin II receptor blocker (ARB).

First, we used a random forest algorithm. ${ }^{13} \mathrm{~A}$ random forest is composed of multiple individual decision trees that operate as an ensemble (online supplementary material). To derive the random forest, we split the data randomly into a $50 \%$ partition used for training and $50 \%$ used for validation. We tuned the number of iterations (ie, number of subtrees) and number of variables to examine randomly at each split. We used 500 iterations to obtain stable out-of-bag and validations errors. We then used the lowest validation error to determine the number of variables in each model. The random forest does not rely on selecting variables, but predictors have different relative importance in the prediction. Importance of a variable was assessed by minimal depth from the tree trunk. We displayed plots of importance score for each predictor.

Second, we applied logistic regression with backward elimination to estimate ORs with $95 \%$ CIs. To account for a relevant increase in a continuous covariate, we reported OR per population SD difference. We selected variables based on the Akaike information criterion, which is equivalent to selecting based on a $\mathrm{p}$ value $<0.1570 .{ }^{14} \mathrm{We}$ forced both age and atrial diameter into the models, as they are clinically important predictors. ${ }^{4}$

For both the random forest and logistic regression, we estimated the $\mathrm{C}$ statistic to assess model discrimination. We assessed calibration as the agreement between predicted and observed probabilities of successful cardioversion in deciles of predicted probabilities using the Hosmer-Lemeshow test.

We performed analyses on complete cases. Among women, 300 of $332(90.4 \%)$ patients were complete cases, and among men, 717 of 790 (90.8\%) patients were 
complete cases. All analyses were performed in Stata V.15.1.

\section{Patient and public involvement}

This study was performed without patient involvement. Patients were not invited to comment on the study design and were not consulted to develop patient relevant outcomes or interpret the results. Patients were not invited to contribute to the writing or editing of the manuscript for readability or accuracy.

\section{RESULTS}

\section{Baseline characteristics and cardioversion success}

During the study period, 332 women and 790 men with persistent AF underwent electrical cardioversion. The median age was 71 years among the women and 67 years among the men. Fewer women (29.8\%) than men (36.9\%) had a history of prior cardioversion and/or RFA ( $\mathrm{p}=0.02)$. Baseline characteristics are summarised in table 1.

Immediate restoration of sinus rhythm failed in 41 $(12.4 \%)$ women and in $56(7.1 \%)$ men $(\mathrm{p}=0.004)$. Electrical cardioversion was successful in 149 women (44.9\%) and 394 men $(49.9 \%)(p=0.13)$. Among the 233 female and 498 male patients without prior cardioversion or RFA, electrical cardioversion was successful in 106 women (45.5\%) and 245 men (49.2\%; $\mathrm{p}=0.35)$. No patients died or were lost to follow up.

\section{Prediction of successful cardioversion using machine learning}

In the final models, the out-of-back and prediction errors were $53.6 \%$ and $41.0 \%$ for the women and $50.4 \%$ and $44.8 \%$ for the men, respectively. Figure 1 shows the relative importance of each variable for the prediction of successful cardioversion in men and in women. Among women, the five most important predictors were age, haemoglobin, eGFR, hypertension, and antiarrhythmic class III drugs. C statistic of the random forest model for women was 0.59 (95\% CI 0.51 to 0.68 ) and the model was well calibrated (Hosmer-Lemeshow $\mathrm{p}=0.94$; figure 2A). The five most important predictors among men were haemoglobin, TSH, eGFR, age, and left atrial diameter. $\mathrm{C}$ statistic of the random forest model for men was 0.56 (95\% CI 0.51 to 0.62 ) and the model was well calibrated (Hosmer-Lemeshow $\mathrm{p}=0.36$; figure 2A).

\section{Prediction of successful cardioversion using logistic regression}

Among women, TSH, diabetes mellitus, and use of betablockers were retained as predictors in the multivariable model. We forced age and atrial diameter into the model. Table 2 shows the ORs for the selected model. The model had moderate discrimination with a $\mathrm{C}$ statistic of 0.60 (95\% CI 0.54 to 0.67 ) and was well calibrated (HosmerLemeshow $\mathrm{p}=0.42$; figure 2B). Among men, backward elimination led to retainment of ventricular ejection fraction below $40 \%$ and use of ACEI or ARB as predictors. Additionally, we forced age and atrial diameter into the
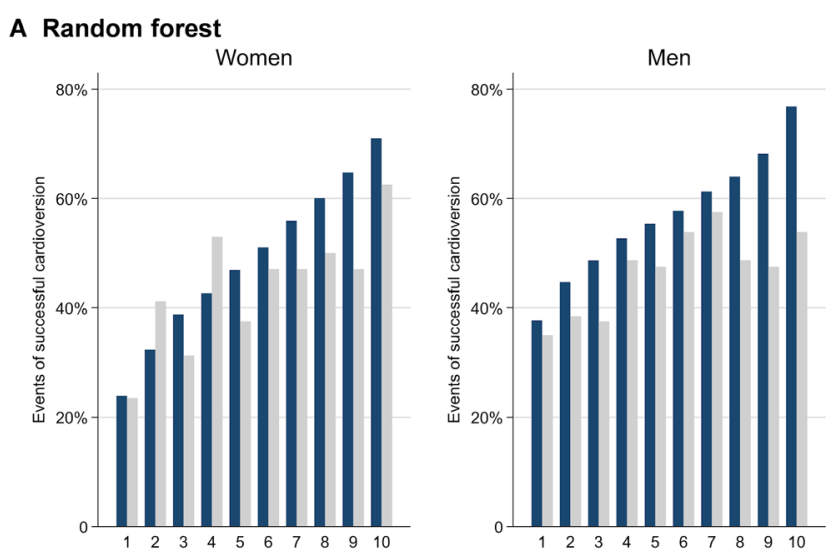

B Logistic regression

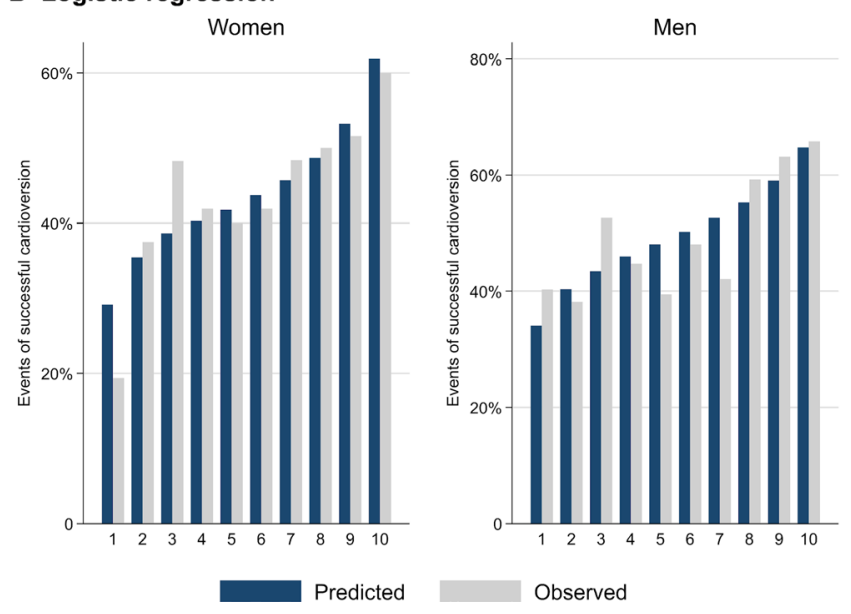

Figure 2 Calibration plots. Agreement between predicted and observed probabilities of successful cardioversion in deciles of predicted odds.

model. The final multivariable prediction model is given in table 2. Like the model among women, the discrimination was moderate, with a C statistic of 0.59 (95\% CI 0.55 to 0.63 ) and the model was well-calibrated well (HosmerLemeshow $\mathrm{p}=0.41$; figure $2 \mathrm{~B}$ ).

\section{Comparison of machine learning and logistic regression predictions}

The five most important variables according to the random forest model were different from the variables selected by the logistic regression model (figure 1 and table 2). Figure 3 shows the distribution of the predicted probabilities of successful electrical cardioversion for the random forest algorithm and logistic regression in men and women. Among the women, the prediction error of the multivariable logistic prediction model was $48.2 \%$ compared with $41.0 \%$ in the random forest algorithm, which corresponded to a difference of 7.2 percentage points in error rate. Among the men, the prediction error of the multivariable logistic prediction model was $46.6 \%$ compared with $44.8 \%$ in the random forest algorithm. This corresponded to a difference of 1.8 percentage points in error rate. 
Table 2 Multivariable logistic regression models for successful cardioversion after electrical cardioversion

OR $(95 \% \mathrm{Cl})^{*}$

\begin{tabular}{|l|l|}
\hline Women & $1.06(0.83$ to 1.35$)$ \\
\hline Age & $0.85(0.67$ to 1.08$)$ \\
\hline Atrial diameter & $1.23(0.96$ to 1.58$)$ \\
\hline Thyroid-stimulating hormone, IU/L & $0.64(0.36$ to 1.15$)$ \\
\hline Beta-blockers & $0.49(0.20$ to 1.22$)$ \\
\hline Diabetes mellitus & \\
\hline Men & $1.05(0.90$ to 1.22$)$ \\
\hline Age & $0.74(0.63$ to 0.87$)$ \\
\hline Left atrial diameter & $1.46(1.02$ to 2.08$)$ \\
\hline LVEF $<40 \%$ (vs $\geq 40 \%)$ & $0.72(0.53$ to 0.97$)$ \\
\hline ACEl or ARB &
\end{tabular}

Among women, 300 of 332 (90.4\%) patients were complete cases, and among men, 717 of $790(90.8 \%)$ patients were complete cases.

*ORs associated with 1 SD increase were reported. SD for women: age 9.91; atrial diameter 0.56; thyroid-stimulating hormone 1.93 . SD for men: age 10.12; atrial diameter 0.57.

ACEI, ACE inhibitor; ARB, angiotensin II receptor blocker; LVEF, left ventricular ejection fraction.

\section{DISCUSSION}

In this large real-world cohort study of consecutive patients with persistent $\mathrm{AF}$, we demonstrated that electrical cardioversion was successful for $45 \%$ of the female patients and $50 \%$ of the male patients during 3 months of follow-up. A higher recurrence rate among women is in accordance with a recent study that demonstrated a higher burden of atrial fibrosis among women with AF, which is associated with structural remodelling and more advanced disease. ${ }^{15}$ The fact that most patients in our study were men may indicate a more conservative clinical approach in the management of women with AF. This tendency is consistent with other studies, in which
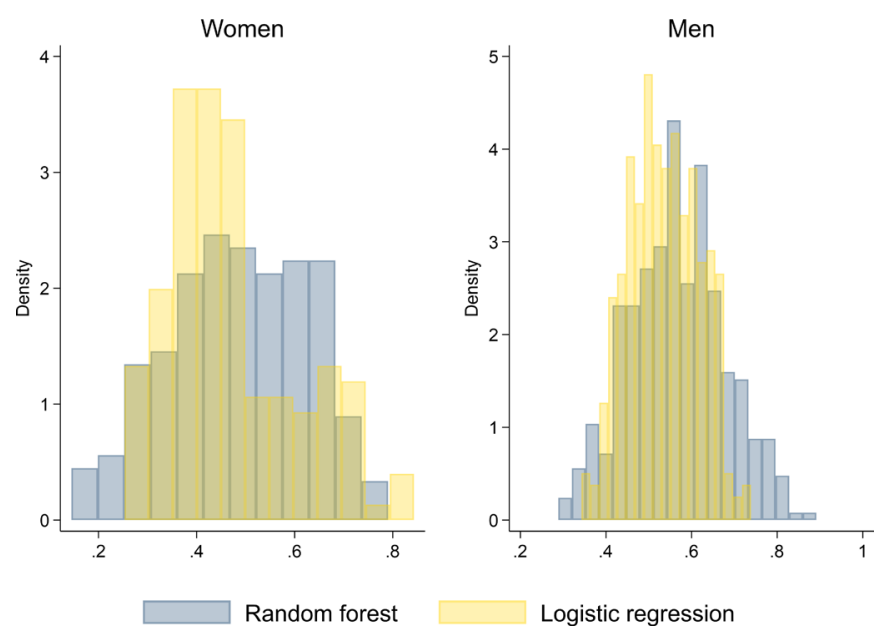

Figure 3 Comparison between machine learning and logistic regression of predicted probabilities of successful electrical cardioversion, by sex. women were scheduled for cardioversion less frequently than men. ${ }^{416}$

We applied two approaches for the development of sexspecific prediction models for successful electrical cardioversion based on several candidate predictors available in routine clinical practice. The random forests and logistic regressions showed only moderate discriminative performance with $\mathrm{C}$ statistics of 0.59 and 0.60 among women, and 0.56 and 0.59 among men, respectively. Based on the clinical factors we included, the ability to predict which patients will benefit from electrical cardioversion is limited for both approaches. Furthermore, our results suggest that there are no substantial differences in the sex-specific predictive performance between the random forest and logistic regression.

Several studies have examined existing prediction models for successful/unsuccessful cardioversion or developed new prediction models. ${ }^{17-21}$ However, we are not aware of any prediction models developed separately in women and men. Accordingly, we cannot compare our findings with the literature directly. Previous studies have supported that atrial diameter is an important predictor. ${ }^{24}$ Contrarily, our random forest analyses did not rank atrial diameter as one of the most important predictors (14th most important predictor among women and 5th most important among men). As we used left atrial diameter as a proxy for atrial size and the left atrium is not uniformly spherical, the atrial diameter may not fully reflect the volume of the left atrium. Volume measurements of the left atrium might be a stronger predictor of ineffective cardioversion than atrial diameter; however, to our knowledge, the predictive ability for successful cardioversion has not been compared between those two measures so far.

Machine learning methods have gained currency in the last years, and a staggered variety of approaches with different properties have been developed. In comparison with typical regression techniques, probably the most prominent property of the decision tree/random forest approach in the present study is the capability of handling high-order interactions between the investigated predictors (as illustrated in the example in the online supplementary material) ${ }^{22}$ Further details about statistical differences have been described elsewhere. ${ }^{22}$ Nevertheless, the presented data held little evidence that the random forest approach provided any meaningful improvement of the prediction of successful cardioversion. This finding agrees well with a recent systematic review, which examined the performance of machine learning compared with logistic regression for the development of prediction models and found no evidence of a superiority of machine learning. ${ }^{23}$

\section{Implications}

Our results suggest that sex-specific identification of patients who will undergo successful cardioversion is challenging in routine clinical practice. Our sex-specific models found different clinically important predictors 
between sexes and approaches, but the models did not demonstrate improved predictive ability compared with other existing models. As our models only demonstrated moderate discrimination, further validation in external cohorts is needed.

Additionally, new approaches for developing prediction models should be considered. In a recent paper by Oto $e t a l$, the authors used a data mining algorithm to identify predictors of recurrence of persistent $\mathrm{AF}^{24}$ To our knowledge, no studies have so far applied data mining algorithms to develop sex-specific prediction models for successful electrical cardioversion. Another future approach may be the use of personalised computational modelling of arrhythmogenesis, which was recently applied among patients with persistent AF to identify ablation targets. ${ }^{25}$ Theoretically, use of personalised computational models of the atria may be used to identify atrial substrate associated with a potential sustained effect of electrical cardioversion.

In contemporary medical practice, the shared decision of an electrical cardioversion needs to include a discussion on the high risk of recurrence of $\mathrm{AF}$ and willingness for exposure to antiarrhythmic drugs and ablation. Randomised trials have shown that rhythm control is not superior to rate control in the management of $\mathrm{AF}^{26} 27$ Interestingly, these trials showed that mortality rates were significantly higher in women randomised to rhythm control as compared with men. Additionally, in relation to outcomes other than mortality, women assigned to rhythm control encountered worse outcomes compared with women in the rate control group. ${ }^{628} 29$ Therefore, since rhythm control is less beneficial in women and associated with more adverse events, patient sex may be important when deciding the optimal treatment strategy in AF.

\section{Limitations}

Our study had important limitations. Selection of patients for electrical cardioversion was based on shared decisions, which may reduce the generalisability of our results. For instance, our cohort included no multimorbid elderly patients and it is possible that patients with very enlarged atria and/or manifest heart failure were not scheduled for cardioversion. However, this way of recruiting patients reflects a real-world clinical setting. Information on other potential important predictors such as duration of AF or electrophysiological data was not available. We were not able to follow the patients with Holter monitoring or a loop/event recorder during the 3 months of follow-up and we had no information on the course of the patients' symptoms.

\section{CONCLUSIONS}

In patients with persistent AF, sex-specific prediction of successful cardioversion is challenging. Machine learning and logistic regression models demonstrated modest predictive performance for successful electrical cardioversion. The high recurrence rate calls for thoroughly informed shared decision-making for electrical cardioversion, which should include a discussion about antiarrhythmic drug therapy and ablation in case of a failed cardioversion.

Contributors NV, ASF, LF, AEA and DSM participated in the original planning, conduct and design of the study; collected the data. NV, MF-G and LT performed the statistical analyses. NV, LT, MF-G and LF drafted the manuscript and ASF, AEA, GYHL and DSM provided manuscript editing and comments and suggestions.

Funding An unrestricted grant from Bristol-Myers Squibb (BMS) and Pfizer supported this study.

Disclaimer The sponsor had no role in the study design, in the collection and interpretation of the data, in the writing of this report, or in the decision to submit the article for publication.

Competing interests AEA: has been on the speaker bureaus for Astra Zenica, Bayer, BMS, Boehringer Ingelheim and Pfizer. GYHL: consultant for Bayer/Janssen, BMS/Pfizer, Medtronic, Boehringer Ingelheim, Novartis, Verseon and Daiichi-Sankyo. Speaker for Bayer, BMS/Pfizer, Medtronic, Boehringer Ingelheim and DaiichiSankyo. No fees are directly received personally. LT: is supported by a grant from AHA (18SFRN34150007). LF: has been an advisory board member for BMS, MSD and Pfizer in relation to non-interventional studies and has been on the speaker bureaus for Bayer, BMS, Boehringer Ingelheim, MSD and Pfizer. DSM: has been on the speaker bureaus for Bayer, BMS, Boehringer Ingelheim, MSD and Pfizer.

Patient consent for publication Not required.

Ethics approval The Danish Data Protection Agency (1-16-02-427-15) and the Medicines Authority (3-3013-1165/1) approved this study. Approval from an Ethics Committee was not required according to Danish law.

Provenance and peer review Not commissioned; externally peer reviewed.

Data availability statement No data are available. Data cannot be made available as access to patient records and public sharing of data are not legal, cf. Danish law.

Open access This is an open access article distributed in accordance with the Creative Commons Attribution Non Commercial (CC BY-NC 4.0) license, which permits others to distribute, remix, adapt, build upon this work non-commercially, and license their derivative works on different terms, provided the original work is properly cited, appropriate credit is given, any changes made indicated, and the use is non-commercial. See: http://creativecommons.org/licenses/by-nc/4.0/.

ORCID iD

Nicklas Vinter http://orcid.org/0000-0003-0558-8483

\section{REFERENCES}

1 Frederiksen AS, Albertsen AE, Christesen AMS, et al. Cardioversion of atrial fibrillation in a real-world setting: non-vitamin $\mathrm{K}$ antagonist oral anticoagulants ensure a fast and safe strategy compared to warfarin. Europace 2018;20:1078-85.

2 Abu-El-Haija B, Giudici MC. Predictors of long-term maintenance of normal sinus rhythm after successful electrical cardioversion. Clin Cardiol 2014;37:381-5.

3 Raitt MH, Volgman AS, Zoble RG, et al. Prediction of the recurrence of atrial fibrillation after cardioversion in the atrial fibrillation follow-up investigation of rhythm management (AFFIRM) study. Am Heart $J$ 2006;151:390-6.

4 Ecker V, Knoery C, Rushworth G, et al. A review of factors associated with maintenance of sinus rhythm after elective electrical cardioversion for atrial fibrillation. Clin Cardiol 2018;41:862-70.

5 Odening KE, Deiß S, Dilling-Boer D, et al. Mechanisms of sex differences in atrial fibrillation: role of hormones and differences in electrophysiology, structure, function, and remodelling. Europace 2019;21:366-76.

6 Rienstra M, Van Veldhuisen DJ, Hagens VE, et al. Gender-related differences in rhythm control treatment in persistent atrial fibrillation: data of the rate control versus electrical cardioversion (RACE) study. J Am Coll Cardiol 2005;46:1298-306.

7 Piccini JP, Simon DN, Steinberg BA, et al. Differences in clinical and functional outcomes of atrial fibrillation in women and men: two-year results from the ORBIT-AF registry. JAMA Cardiol 2016;1:282-91.

8 Linde C, Bongiorni MG, Birgersdotter-Green U, et al. Sex differences in cardiac arrhythmia: a consensus document of 
the European heart rhythm association, endorsed by the heart rhythm Society and Asia Pacific heart rhythm Society. Europace 2018;20:1565-1565ao.

9 Krittanawong C, Johnson KW, Rosenson RS, et al. Deep learning for cardiovascular medicine: a practical primer. Eur Heart $J$ 2019;40:2058-73.

10 Oikonomou EK, Williams MC, Kotanidis CP, et al. A novel machine learning-derived radiotranscriptomic signature of perivascular fat improves cardiac risk prediction using coronary CT angiography. Eur Heart J 2019;40:3529-43.

11 Motwani M, Dey D, Berman DS, et al. Machine learning for prediction of all-cause mortality in patients with suspected coronary artery disease: a 5-year multicentre prospective registry analysis. Eur Heart J 2016;124:ehw188-7.

12 Kirchhof P, Benussi S, Kotecha D, et al. 2016 ESC guidelines for the management of atrial fibrillation developed in collaboration with EACTS. Eur Heart J 2016;37:2893-962.

13 Schonlau M, Zou R, Schonlau M. The random forest algorithm for statistical learning with applications in Stata. Stata Journal 2018;20:3-29.

14 Heinze G, Wallisch C, Dunkler D. Variable selection - A review and recommendations for the practicing statistician. Biom $J$ 2018;60:431-49.

15 Akoum N, Mahnkopf C, Kholmovski EG, et al. Age and sex differences in atrial fibrosis among patients with atrial fibrillation. Europace 2018;20:1086-92.

16 Gurevitz OT, Varadachari CJ, Ammash NM, et al. The effect of patient sex on recurrence of atrial fibrillation following successful direct current cardioversion. Am Heart J 2006;152:155.e9-155.e13.

17 Falsetti L, Viticchi G, Tarquinio N, et al. CHA2DS2-VASc in the prediction of early atrial fibrillation relapses after electrical or pharmacological cardioversion. J Cardiovasc Med 2014;15:636-41.

18 Mlodawska E, Tomaszuk-Kazberuk A, Lopatowska P, et al. CHA DS VASc score predicts unsuccessful electrical cardioversion in patients with persistent atrial fibrillation. Intern Med J 2017;47:275-9.
19 de Vos CB, Pisters R, Nieuwlaat R, et al. Progression from paroxysmal to persistent atrial fibrillation clinical correlates and prognosis. J Am Coll Cardiol 2010;55:725-31.

20 Lankveld T, de Vos CB, Limantoro I, et al. Systematic analysis of ECG predictors of sinus rhythm maintenance after electrical cardioversion for persistent atrial fibrillation. Heart Rhythm 2016;13:1020-7.

21 Jaakkola S, Lip GYH, Biancari F, et al. Predicting unsuccessful electrical cardioversion for acute atrial fibrillation (from the AF-CVS score). Am J Cardiol 2017;119:749-52.

22 Boulesteix A-L, Schmid M. Machine learning versus statistical modeling. Biom J 2014;56:588-93.

23 Christodoulou E, Ma J, Collins GS, et al. A systematic review shows no performance benefit of machine learning over logistic regression for clinical prediction models. J Clin Epidemiol 2019;110:12-22.

24 Oto E, Okutucu S, Katircioglu-Öztürk D, et al. Predictors of sinus rhythm after electrical cardioversion of atrial fibrillation: results from a data mining project on the Flec-SL trial data set. Europace 2017;19:921-8.

25 Boyle PM, Zghaib T, Zahid S, et al. Computationally guided personalized targeted ablation of persistent atrial fibrillation. Nat Biomed Eng 2019;3:870-9.

26 Wyse DG, Waldo AL, DiMarco JP, et al. A comparison of rate control and rhythm control in patients with atrial fibrillation. $N$ Engl $\mathrm{J} M e d$ 2002;347:1825-33.

27 Van Gelder IC, Hagens VE, Bosker HA, et al. A comparison of rate control and rhythm control in patients with recurrent persistent atrial fibrillation. N Engl J Med 2002;347:1834-40.

28 Curtis AB, Gersh BJ, Corley SD, et al. Clinical factors that influence response to treatment strategies in atrial fibrillation: the atrial fibrillation follow-up investigation of rhythm management (AFFIRM) study. Am Heart J 2005;149:645-9.

29 Kaufman ES, Zimmermann PA, Wang T, et al. Risk of proarrhythmic events in the atrial fibrillation follow-up investigation of rhythm management (AFFIRM) study: a multivariate analysis. J Am Coll Cardiol 2004;44:1276-82. 